\title{
Clinical outcome of extended-field irradiation vs. pelvic irradiation using intensity-modulated radiotherapy for cervical cancer
}

\author{
YI OUYANG $^{1 *}$, YANHONG WANG $^{2 *}, \mathrm{KAI} \mathrm{CHEN}^{1}, \mathrm{XINPING} \mathrm{CAO}^{1}$ and YIMING ZENG $^{3}$ \\ ${ }^{1}$ Department of Radiotherapy, Sun Yat-Sen University Cancer Center, State Key Laboratory of Oncology in South China, \\ Collaborative Innovation Center for Cancer Medicine, Guangzhou, Guangdong 510060; ${ }^{2}$ Department of Radiotherapy, \\ ${ }^{3}$ Respiratory Department, The Second Affiliated Hospital of Fujian Medical University, Quanzhou, Fujian 362000, P.R. China
}

Received July 22, 2016; Accepted July 11, 2017

DOI: $10.3892 / \mathrm{ol} .2017 .7077$

\begin{abstract}
The aim of the present study was to evaluate the distinctions in survival and toxicity between patients with cervical cancer with common iliac node or para-aortic node involvement, who were treated with extended-field intensity-modulated radiotherapy (EF-IMRT) and patients with or without lower involved pelvic nodes, who were treated with pelvic IMRT. A total of 55 patients treated with EF-IMRT and 52 patients treated with pelvic IMRT at the Sun Yat-Sen University Cancer Center (Guangzhou, China) were retrospectively analyzed. Patients treated with EF-IMRT had the highest level of lymph node involvement to the para-aortic or common iliac nodes, while patients treated with pelvic IMRT had no para-aortic or common iliac nodes involved $(\mathrm{P}<0.001)$. The median follow-up time was 29.5 months. The 3-year overall survival (OS) rates of EF-IMRT and pelvic IMRT were 79.4 and $82.3 \%(\mathrm{P}=0.45)$, respectively, and the 3-year disease-free survival (DFS) rates of EF-IMRT and pelvic IMRT were 61.0 and $73.7 \%(\mathrm{P}=0.55)$, respectively. Cox's regression analysis revealed that EF irradiation was a protective prognostic factor for OS and DFS. A total of 16 patients in the EF-IMRT group and 13 patients in the pelvic IMRT group experienced treatment failure $(\mathrm{P}=0.67)$, with the patterns of failure being the same for the two groups $(\mathrm{P}=0.88)$. The cumulative incidence of grade 3 and 4 acute toxicities in the EF-IMRT group was $34.5 \%$,
\end{abstract}

Correspondence to: Professor Xinping Cao, Department of Radiotherapy, Sun Yat-Sen University Cancer Center, State Key Laboratory of Oncology in South China, Collaborative Innovation Center for Cancer Medicine, 651 Dongfeng East Road, Guangzhou, Guangdong 510060, P.R. China

E-mail: caoxp@sysucc.org.cn

Dr Yiming Zeng, Respiratory Department, The Second Affiliated Hospital of Fujian Medical University, 34 Zhongshan North Street, Quanzhou, Fujian 362000, P.R. China

E-mail: zeng_yi_ming@126.com

*Contributed equally

Key words: cervical cancer, intensity-modulated radiotherapy, extended-field radiotherapy, pelvic radiotherapy, survival, toxicity in comparison with $19.2 \%$ in the pelvic group $(\mathrm{P}=0.048)$. The results of the present study suggest that patients with cervical cancer with grossly involved common iliac or para-aortic nodes should be electively subjected to EF irradiation to improve the survival and alter patterns of recurrence. Notably, EF irradiation delivered via IMRT exhibits an increased toxicity incidence, however, this remains within an acceptable range.

\section{Introduction}

Concurrent cisplatin-based chemoradiation is considered the standard treatment for locally advanced cervical cancer, with the aim of improving local control and overall survival (OS). However, Lin et al (1) identified that a sizeable proportion of patients with cervical cancer exhibit para-aortic lymph node (PALN) involvement, with $14 \%$ of International Federation of Gynecology and Obstetrics stage (2) I, 20\% of stage II and $32 \%$ of stage III cancer cases involved with the para-aortic lymph nodes. Thus, patients treated with standard pelvic field irradiation possess shortened 5-year disease-free survival (DFS) times (3). The Radiation Therapy Oncology Group (RTOG) 79-20 trial demonstrated that prophylactic para-aortic irradiation using anteroposterior fields may improve OS and decrease distant metastasis for patients with cervical cancer with bulky tumors or stage IIB disease (4). However, the cumulative incidence of grade 4 and 5 toxicities, and the proportion of mortalities as a result of radiotherapy complications are considerably increased for EF irradiation compared with pelvic irradiation (4). Therefore, the therapeutic value of prophylactic irradiation for PALNs remains unclear in conventional radiotherapy.

Although intensity-modulated radiotherapy (IMRT) is used to decrease toxicity, treatment of the para-aortic nodal regions has inherent toxicity. Furthermore, cervical cancer appears to exhibit an orderly pattern of spread, initially involving the low pelvic lymph nodes prior to progressing to the high pelvic lymph nodes and PALNs (3). In addition, IMRT costs are covered by national insurance in China, which decreases the financial burden on patients. Therefore, since January 2011, EF-IMRT has been electively performed for patients with cervical cancer with common iliac node or PALN involvement, and pelvic IMRT has been electively performed for patients with or without low pelvic nodal metastasis. Overall, 
the aim of the present study was to evaluate the efficacy and toxicities of EF-IMRT vs. pelvic IMRT.

\section{Materials and methods}

Patients. A total of 181 patients (mean age, 56.3 years; range, 27-83) with cervical squamous-cell cancer who were treated with IMRT and high-dose-rate brachytherapy (HDR BT) at the Sun Yat-Sen University Cancer Center (Guangzhou, China) between March 2011 and May 2013 were retrospectively reviewed. Among them, 74 patients were excluded for meeting any one of the following criteria: i) Stage IVB disease; ii) distant nodal metastasis in inguinal, mediastinal or supraclavicular lymphatics; iii) treatment with salvage, palliative or adjuvant intent; or iv) incomplete radiotherapy due to patient refusal or poor performance status. The Institutional Review Board of the Sun Yat-Sen University Cancer Center approved the present study.

Among the remaining patients, 55 and 52 patients were treated with EF-IMRT and pelvic IMRT, respectively. All patients underwent abdominal and pelvic magnetic resonance imaging (MRI) to assess nodal metastasis and tumor size. As a result of economic factors, the majority of patients underwent a chest X-ray and color ultrasound of the supraclavicular region to evaluate the distant metastasis instead of a positron emission tomography (PET) and chest computed tomography $(\mathrm{CT})$ scan. The patient characteristics of the two groups are presented (Table I).

Treatment. All patients were treated with a combination of IMRT and HDR BT. The IMRT process incorporated CT-based simulation, performed with a full bladder and an empty rectum, and each patient underwent a planning CT scan from the upper border of T10 to the ischialtuberosity, with a 3-mm slice thickness (Philips Medical Systems, Inc., Bothell, WA, USA). The target volumes and organs at risk, including the spinal cord, bowel, kidneys, bladder, rectum and femoral heads, were drawn on each planning CT slice (5). Involved lymph nodes (a short-axis diameter on CT/MRI of $>1 \mathrm{~cm}$ ) were contoured as gross tumor volume (GTV-N). The cervical tumor and uterus were contoured as high-risk CTV (HR-CTV), and the doses were increased in BT, but not in external-beam radiotherapy.

Pelvic irradiation. The clinical target volume (CTV) included all areas of gross and potentially microscopic diseases. The pelvic CTV consisted of a 0.7 to $2-\mathrm{cm}$ margin around the vessels, cervix, uterus, parametria, presacral space and vagina (6). The inguinal region was drawn as part of the CTV when the distal vagina or inguinal lymph nodes was considered to be involved, according to imaging and clinical examination.

EF irradiation. Patients with evidence for positive involvement of para-aortic or high common iliac nodes were treated with EFR to the superior border of the first lumbar vertebra. The CTV in the para-aortic region was contiguous with the pelvic lymph node stations and encompassed the aorta and inferior vena cava with a $1-$ to $1.5-\mathrm{cm}$ minimum margin (7).

The GTV-N was expanded by $0.5 \mathrm{~cm}$ to create the planning target volume of the GTV-N (PTVGTV-N) and the CTV was expanded by $0.6-1 \mathrm{~cm}$ to create the PTVCTV, accounting for patient motion and set-up uncertainty. The CTV dose was 45 Gy in 25 fractions, with a concomitant boost of GTV-N to a dose of 60 Gy in 25 fractions (Fig. 1A and B).

HDR BT. The median BT dose was 36 Gy in 6 fractions to the periphery of the HR-CTV, with a combined intracavitary/interstitial technique. The prescribed dose to the periphery of the HRCTV was 6 Gy. The radiation source $\left({ }^{192} \mathrm{Ir}\right)$ dwell time was adjusted using graphic optimization until the dose-volume constraints were optimally matched (Fig. 2). The planning aimed to deliver a minimum of 85 Gy to $90 \%$ of the HR-CTV in 2 Gy equivalent (EqD2), adding BT and external beam radiotherapy doses, and applying the linear quadratic model with an $\alpha / \beta$ ratio of $10 \mathrm{~Gy}$.

Chemotherapy. It was planned that the patients would receive cisplatin $\left(40 \mathrm{mg} / \mathrm{m}^{2}\right)$ via intravenous infusion every week for 6 weeks during the course of external beam radiotherapy, and that patients with the following conditions would not receive concurrent chemotherapy: i) Elderly age ( $>70$ years); ii) Performance Status score $>2$ (8); or iii) rejection of chemotherapy. Complete blood count tests were performed weekly and chemotherapy was withheld until resolution to at least grade 1 if patients presented with grade 3 or 4 hematological or gastrointestinal toxicity.

Follow-up. All patients were examined at 1 month post-radiotherapy, every 3 months during the first and second years, and every 6 months in the third year of follow-up. Follow-up investigations included clinical examination, Papanicolau smears, serum tumor marker (squamous cell carcinoma antigen) analysis and cross-sectional imaging (pelvic MRI and abdominal CT). Chest CT examination, supraclavicular lymph node ultrasound examination and bone scintigraphy were performed once a year to evaluate distant recurrence (lung, supraclavicular nodes and bone).

Follow-up abdominal CT and pelvic MRI were performed between 1 and 2 months after the completion of radiotherapy to evaluate the response to therapy. Complete response was defined as images and clinical examination resultsidentifying no evidence of local or regional nodal disease. Partial response was defined as any persistence of tumor at the site of local or regional nodes on the axial scan within 3 to 6 months after completion of radiotherapy. Local failure was defined as the recurrence or residual disease at the cervix, uterus or adjacent pelvic organs, e.g., parametria, bladder and vagina. Regional nodal relapse was defined as residual or recurrent, cancer in the pelvic or para-aortic lymph nodes (if the distal vagina was involved, inguinal lymph nodes were also considered regional relapse; otherwise, the nodes were considered distant metastases). Distant failure was defined as recurrence in non-regional lymph nodes (mediastinal and supraclavicular region) or hematogenous metastasis (including in the bones, liver and lungs). Failure was recorded on the basis of clinical examination and follow-up imaging (MRI/CT or PET/CT), and the majority of patients received biopsy confirmation.

Acute toxicity associated with radiotherapy was assessed in accordance with the RTOG acute radiation morbidity scoring criteria (9) and was defined as toxicity occurring 
Table I. Patient characteristics for extended-field irradiation and pelvic irradiation.

\begin{tabular}{|c|c|c|c|}
\hline Characteristics & Extended-field $(\mathrm{n}=55)$ & Pelvic $(\mathrm{n}=52)$ & P-value \\
\hline Mean age (range) & $55.1(27-80)$ & $59.4(38-83)$ & 0.048 \\
\hline FIGO stage, $\mathrm{n}$ & & & $0.82^{\mathrm{b}}$ \\
\hline I & 4 & 5 & \\
\hline II & 28 & 28 & \\
\hline III & 23 & 19 & \\
\hline Tumor size, $\mathrm{cm}^{\mathrm{a}}$ & $4.95 \pm 1.57$ & $4.55 \pm 1.80$ & 0.21 \\
\hline Vaginal invasion, $\mathrm{n}$ & & & $0.59^{\mathrm{b}}$ \\
\hline None & 7 & 7 & \\
\hline Upper $1 / 3$ & 33 & 34 & \\
\hline Middle 1/3 & 9 & 9 & \\
\hline Distal 1/3 & 6 & 2 & \\
\hline Uterus invasion, $\mathrm{n}$ & & & $0.12^{\mathrm{b}}$ \\
\hline No & 19 & 26 & \\
\hline Yes & 36 & 26 & \\
\hline Highest level of involved lymph nodes, $n$ & & & $<0.001^{\mathrm{b}}$ \\
\hline None & 0 & 27 & \\
\hline Lower pelvic nodes & 0 & 25 & \\
\hline Common iliac nodes & 44 & 0 & \\
\hline PALN & 11 & 0 & \\
\hline Nadir-Hgb, g/1 ${ }^{a}$ & $96.9 \pm 20.6$ & $100.3 \pm 16.1$ & 0.36 \\
\hline Cumulative dose of weekly cisplatin, $\mathrm{mg} / \mathrm{m}^{2 \mathrm{a}}$ & $128.8 \pm 106.8$ & $130.8 \pm 120.2$ & 0.93 \\
\hline Treatment duration, days ${ }^{\mathrm{a}}$ & $69.2 \pm 22.2$ & $63.4 \pm 19.9$ & 0.16 \\
\hline Total dose of D90 (EQD2, Gy) ${ }^{\mathrm{a}}$ & $94.7 \pm 7.3$ & $95.7 \pm 7.1$ & 0.44 \\
\hline Tumor response, $\mathrm{n}$ & & & $0.89^{\mathrm{b}}$ \\
\hline $\mathrm{CR}$ & 45 & 42 & \\
\hline PR & 10 & 10 & \\
\hline
\end{tabular}

${ }^{a}$ Data are presented as the mean \pm standard deviation. ${ }^{b} \chi^{2}$ test. Hgb, hemoglobin; FIGO, International Federation of Gynecology and Obstetrics; $\mathrm{CR}$, complete response; PR, partial response; EQD2, equivalent dose of $2 \mathrm{~Gy}$.
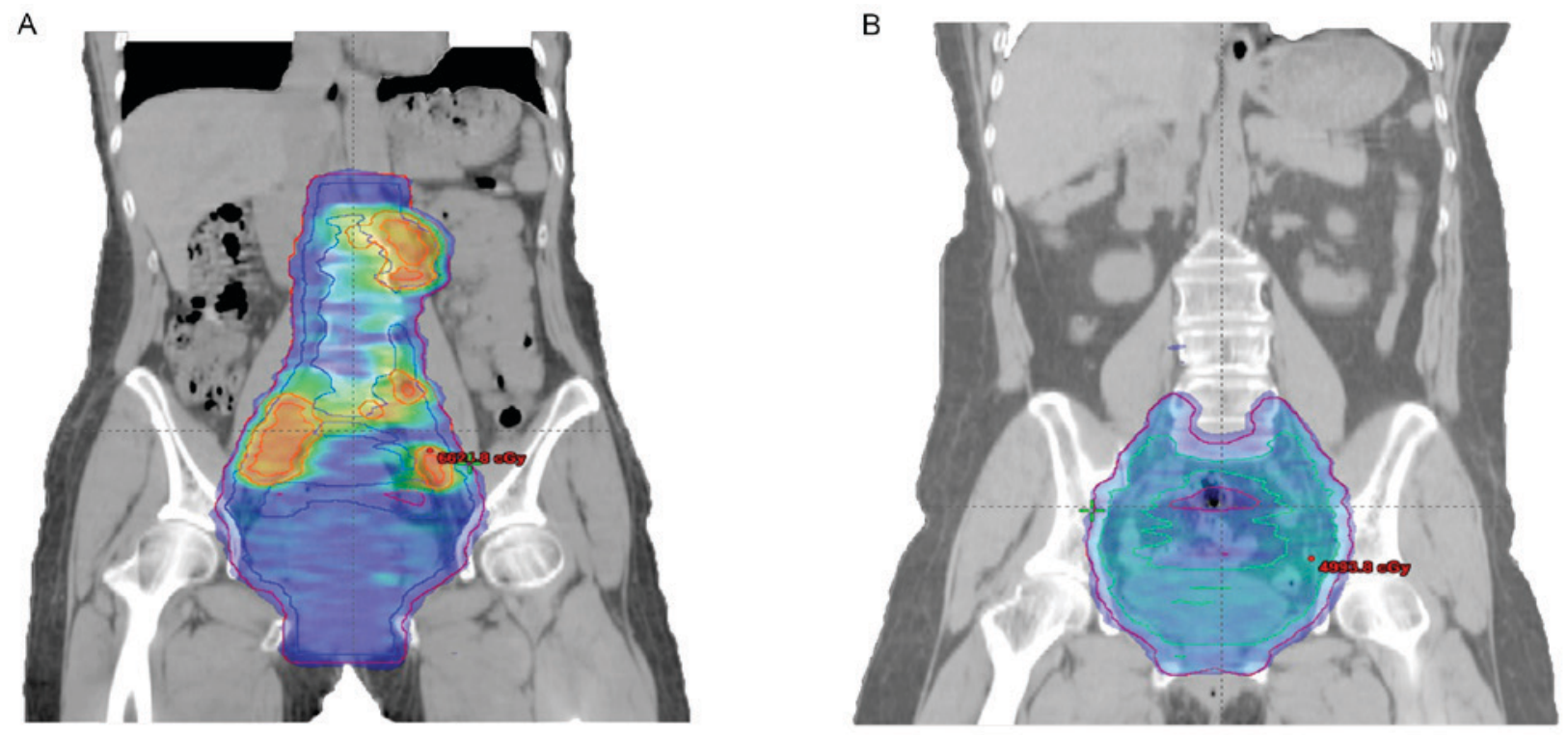

Figure 1. CT images captured following external-beam radiotherapy dosage. Coronal image of external-beam radiotherapy detailing planning target volume (blue line) covered with blue isodose color wash $(45 \mathrm{~Gy})$, with concomitant boost to the involved lymph nodes (red line) covered with orange color wash (60 Gy), and different irradiation fields to the para-aortic lymph nodes: (A) Extended-field radiotherapy and (B) pelvic radiotherapy. CT, computed tomography. 

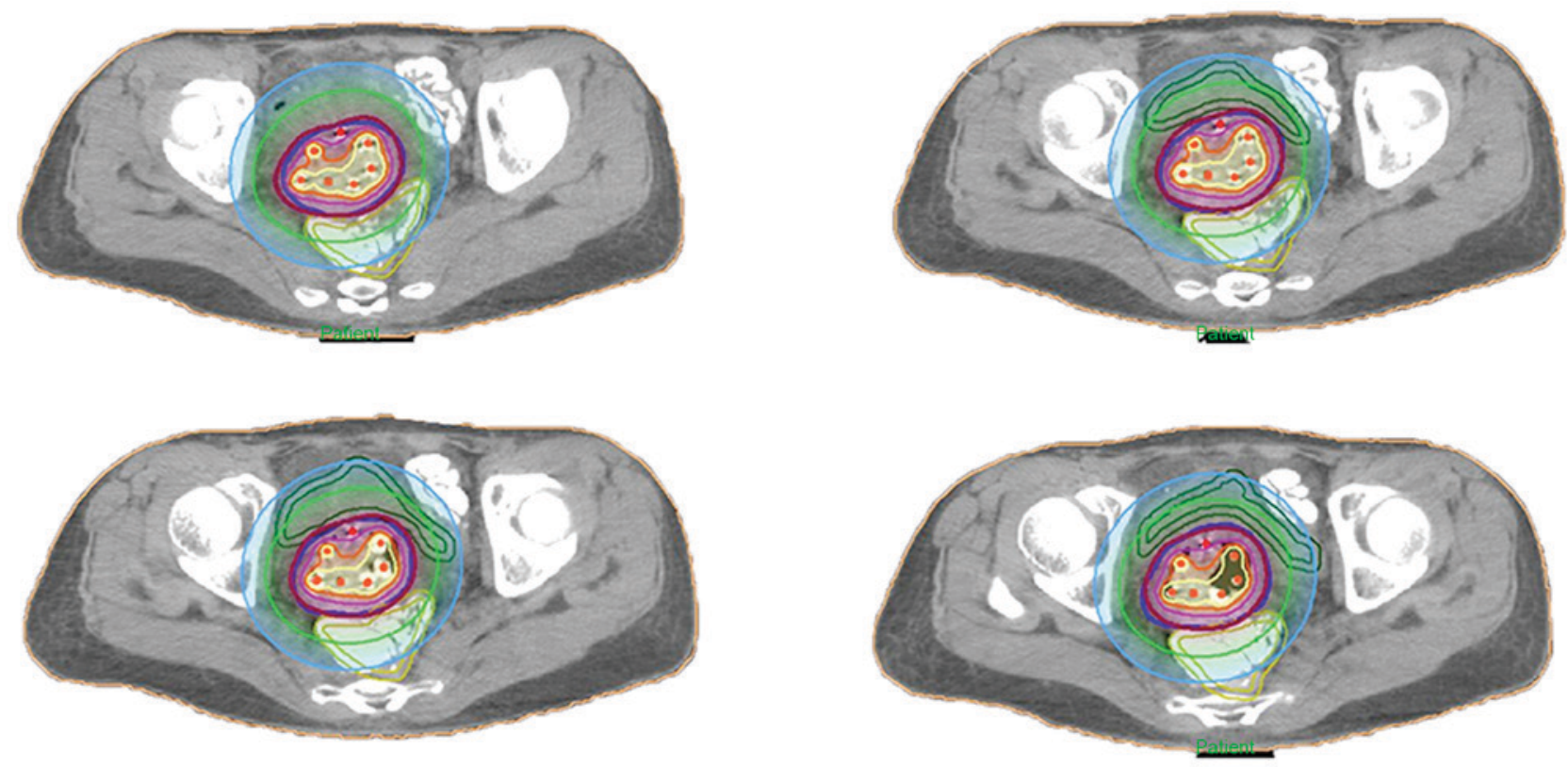

Figure 2. CT images captured following brachytherapy dosage. Axial image of brachytherapy detailing high-risk clinical target (dark blue line) covered with a purple line indicating 6 Gy in 1 fraction for 1 patient with different slices. The dose decreases with distance from the centre, as follows: 12 Gy (yellow line), 10 Gy (orange line), 4 Gy (green line) and 2 Gy (light blue line). CT, computed tomography.

between the initiation of treatment and 90 days after completion. Acute toxicity was assessed weekly during the course of radiotherapy, at the completion of $\mathrm{RT}$ and after 1 month; late effects were evaluated at each clinical visit. Adverse events for $>90$ days after the completion of treatment were graded in accordance with the RTOG late radiation morbidity scoring system (10).

Statistical analysis. Survival time and time to recurrence were measured from the date of initial radiation treatment. An unpaired t-test or $\chi^{2}$ test was used to analyze the associations between patient characteristics, recurrence patterns and toxicities. The Kaplan-Meier estimator method was used to derive estimates of survival. Differences in OS and DFS were assessed using the log-rank test. OS time was calculated from the date of RT start to the date of mortality from any cause or last follow-up. The DFS time was calculated from the date of RT start to the date of disease progression, relapse or initiation of any new, unplanned, anticancer therapies associated with the disease. Disease-associated significant variables on univariate analysis were utilized for Cox's regression analysis to control the confounding factors for OS and DFS. $\mathrm{P}<0.05$ was considered to indicate a statistically significant difference for all study outcomes. All statistical analyses were performed using SPSS (version 17.0; SPSS, Inc., Chicago, IL, USA). Results are presented as the mean \pm standard deviation.

\section{Results}

Survival. The total length of time for the two treatments ranged from 49 to 104 days (median, 61 days). The median duration of follow-up was 29.5 months (range, 7.4 to 50.6 months). A total of 19 out of the 107 patients included in the present study succumbed, including 5 of whom succumbed to concomitant disease (1 cerebral infarction, 1 heart failure, 1 myocardial

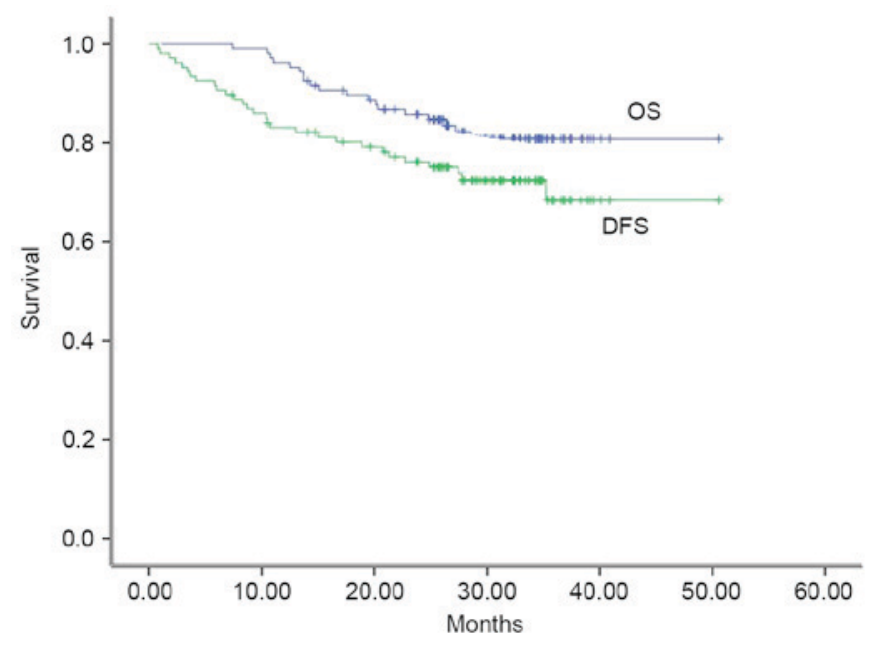

Figure 3. Kaplan-Meier estimator curve of OS and DFS. OS, overall survival; DFS, disease-free survival.

infarction and 2 severe pulmonary infections). The mean survival time was 46.1 months, and the 2- and 3 -year OS rates were 87.7 and $80.7 \%$, respectively. A total of 29/107 patients (27.1\%) exhibited failure at either local, regional node or distant sites. The mean DFS time was 41.2 months, and the 2- and 3-year DFS rates were 77.1 and $72.4 \%$, respectively. The OS and DFS rates are presented (Fig. 3).

A total of 11 out of the 55 patients treated with EF-IMRT succumbed. The mean survival time was $35.0 \pm 1.40$ months, and the 2- and 3-year OS rates were 81.5 and $79.4 \%$, respectively. A total of 16 patients developed recurrence and the mean DFS was 31.2 \pm 1.93 months. The 2- and 3-year DFS rates were 73.7 and $61.0 \%$, respectively.

A total of 8 patients treated with pelvic IMRT succumbed during the follow-up period. The mean survival time for 
A

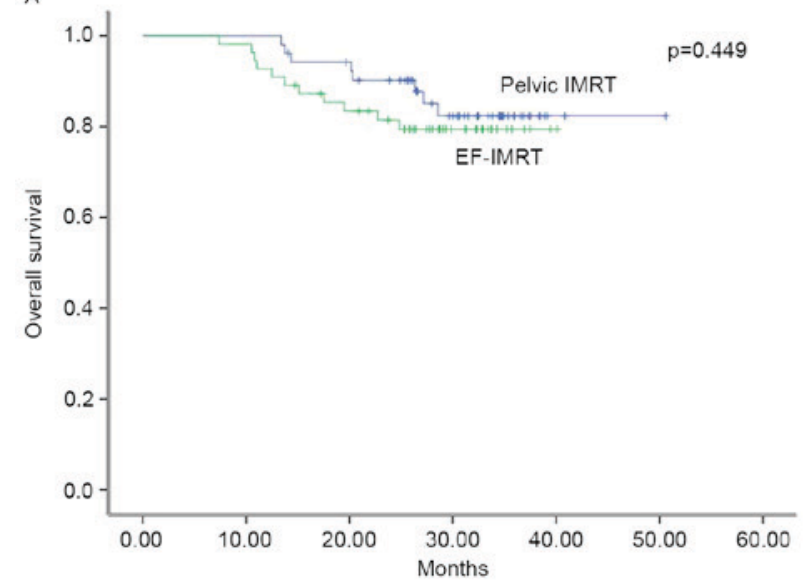

B

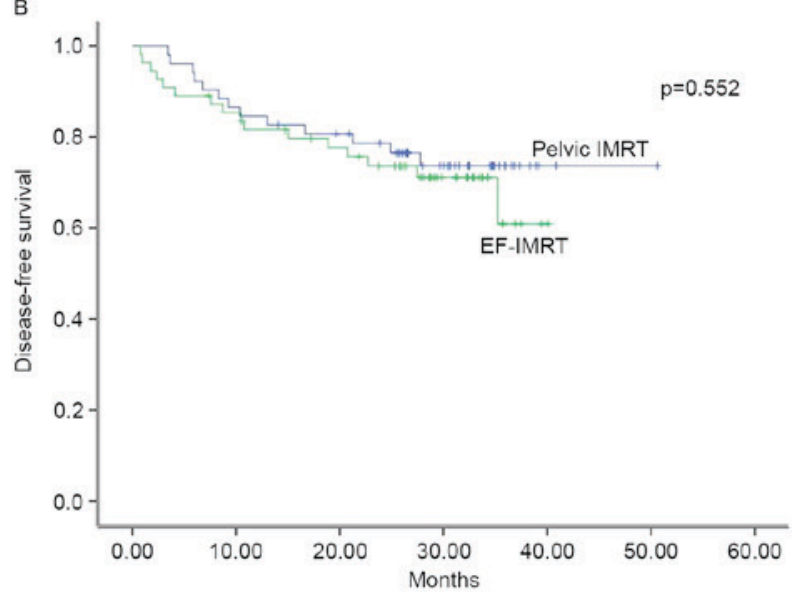

Figure 4. Kaplan-Meier estimator curves for: (A) Overall survival of patients undergoing EF-IMRT and pelvic IMRT, and (B) disease-free survival of patients undergoing EF-IMRT and pelvic IMRT. EF-IMRT, extended-field intensity-modulated radiotherapy.

pelvic irradiation was $45.4 \pm 1.67$ months, and the 2 - and 3 -year OS rates were 90.2 and $82.3 \%$, respectively. A total of 13 patients experienced failure and the mean DFS time was $40.7 \pm 2.42$ months. The 2- and 3-year DFS rates for patients treated with pelvic IMRT were 78.3 and $73.7 \%$, respectively. The differences in OS and DFS between EF-IMRT and pelvic IMRT patients were assessed using the log-rank test and no statistically significant differences were identified (Fig. 4A and B).

The univariate analysis revealed that patients with bulky tumors, a decreased nadir for hemoglobin concentration, increased treatment duration or increased level of LN metastasis were associated with diminished survival rates (Table II). The irradiation fields (EFor pelvic field) were entered and significant variables on univariate analysis were utilized for Cox's regression analysis to evaluate the effect of irradiation field on survival (Table III). It was revealed that patients treated with EF irradiation exhibited an improved prognosis compared with those treated with pelvic field when confounding factors, including the nadir-hemoglobin level, the most distant level of lymph nodes involved, and treatment duration, were controlled.

Recurrent patterns. Of the 16 patients treated with EF-IMRT and who developed recurrence, 7 patients presented with more than one site of failure. A total of 7 of the 55 EF-IMRT patients $(12.7 \%)$ developed local failure, 6 (10.9\%) patients developed regional relapse (3 developed in para-aortic nodes) and 9 (16.4\%) patients exhibited distant metastasis. Among the patients treated with pelvic IMRT, 13 patients relapsed and 4 patients presented more than one site of failure. A total of 6 of the 52 pelvic IMRT patients (11.5\%) developed local failure, 3 (5.8\%) developed regional failures (2 developed in para-aortic nodes) and 6 (11.5\%) exhibited distant metastasis. No significant differences in the failure rate or proportion of recurrent sites between the two groups were identified in the patients $(\mathrm{P}=0.67$ and $\mathrm{P}=0.88$, respectively).

Toxicities. Acute toxicities are presented in Table IV. The two treatments were well-tolerated, with 19 (34.5\%) and $10(19.2 \%)$ patients experiencing grade 3 or greater acute toxicities for
EF-IMRT and pelvic IMRT, respectively ( $\mathrm{P}=0.048$, Fisher's exact test). For EF-IMRT, 2 patients experienced severe nausea or vomiting, and 3 patients experienced severe diarrhea, which required pharmacological intervention. Of the 14 patients with grade 3 or greater hematological toxicities, 4 patients developed anemia and required a blood transfusion, and 10 patients with grade 3 or 4 leukopenia also presented with neutropenia. Of the patients treated with pelvic IMRT, 2 patients exhibited severely altered bowel habits, 2 possessed nadir-hemoglobin $<70 \mathrm{~g} / \mathrm{l}$ (normal range, 110-130 g/l) and 5 patients developed grade 3 leukopenia accompanied with neutropenia.

As for late toxicities, 5 patients treated with EF-IMRT and 4 treated with pelvic IMRT experienced grade 3 rectal bleeding requiring transfusion or surgery, and 1 patient treated with pelvic IMRT was diagnosed with grade 4 rectovaginal fistula as a result of local recurrence.

\section{Discussion}

Salama et al (10) reported that IMRT planning improves dosimetry, decreases the volume of normal tissue irradiated, and exhibits favorable local control and decreased acute gastrointestinal, genitourinary and bone marrow toxicities in comparison with conventional treatment. EFRT is conventionally indicated for patients with cervical cancer with grossly detected common iliac or PALN metastasis on the basis of orderly spread patterns and decreased rates of skip metastasis (11). Therefore, patients with cervical cancer in the Sun Yat-Sen University Cancer Center received EF-IMRT when common iliac nodes or PALN were involved, and pelvic IMRT when negative nodes or lower pelvic nodes were involved.

Wu et al (12) retrospectively analyzed the data of 55 patients with PALN-positive cervical cancer treated with (27 patients) or without (28 patients) EFRT, and revealed that the 3 -year OS rates were $\sim 50$ and $23 \%$, respectively (12). The results from another study on 39 patients with grossly involved common iliac nodes or PALN treated with EFRT demonstrated that the 3-year OS and DFS rates were 45 and $23 \%$ (13), which is inferior to the patients in the current study treated with EFRT (79.4 and 61.1\%). In the aforementioned 
Table II. Univariate analysis for OS and DFS.

\begin{tabular}{|c|c|c|c|c|c|c|}
\hline \multirow[b]{2}{*}{ Characteristics } & \multicolumn{3}{|c|}{ 3-year OS } & \multicolumn{3}{|c|}{ 3-year DFS } \\
\hline & $\mathrm{RR}$ & $95 \% \mathrm{CI}$ & P-value & $\mathrm{RR}$ & $95 \% \mathrm{CI}$ & P-value \\
\hline Tumor size & 1.323 & $1.021-1.713$ & 0.034 & 1.340 & $1.078-1.666$ & 0.008 \\
\hline Nadir-Hgb & 0.976 & 0.954-0.999 & 0.039 & 0.975 & $0.956-0.993$ & 0.008 \\
\hline Treatment duration & 1.024 & $1.009-1.041$ & 0.003 & 1.016 & $1.001-1.030$ & 0.031 \\
\hline Level of LN involved & 1.322 & $1.150-1.520$ & 0.042 & 1.240 & $1.114-1.381$ & 0.045 \\
\hline Irradiation field (extended vs. pelvic) & 1.419 & $0.571-3.529$ & 0.451 & 1.248 & $0.600-2.597$ & 0.553 \\
\hline
\end{tabular}

OS, overall survival; DFS, disease-free survival; Hgb, hemoglobin; LN, lymph nodes; CI, confidence interval; RR, relative risk.

Table III. Cox's regression for 3-year OS and DFS.

\begin{tabular}{|c|c|c|c|c|c|c|}
\hline \multirow[b]{2}{*}{ Characteristics } & \multicolumn{3}{|c|}{ 3-year OS } & \multicolumn{3}{|c|}{ 3-year DFS } \\
\hline & RR & $95 \% \mathrm{CI}$ & P-value & RR & $95 \% \mathrm{CI}$ & P-value \\
\hline Tumor size & 1.623 & $1.238-2.127$ & 0.024 & 1.276 & $1.017-1.601$ & 0.036 \\
\hline The nadir Hgb & 0.983 & 0.970-0.0996 & 0.016 & 0.977 & $0.958-0.997$ & 0.025 \\
\hline The level of LN metastasis & 1.795 & $1.554-2.073$ & 0.039 & 1.510 & $1.353-1.685$ & 0.005 \\
\hline $\begin{array}{l}\text { Irradiation field }^{\mathrm{a}} \\
\text { (extended vs. pelvic) }\end{array}$ & 0.767 & $0.590-0.996$ & 0.011 & 0.874 & $0.783-0.976$ & 0.043 \\
\hline Treatment duration & 1.024 & $1.008-1.041$ & 0.003 & 1.006 & $0.984-1.028$ & 0.094 \\
\hline
\end{tabular}

${ }^{a}$ Variates are entered into Cox models for OS and DFS. DFS, disease-free survival; Hgb, hemoglobin; LN, lymph nodes; OS, overall survival; $\mathrm{CI}$, confidence interval; RR, relative risk.

Table IV. Acute toxicities according to the Radiation Therapy Oncology Group acute radiation morbidity scoring criteria.

\begin{tabular}{|c|c|c|c|c|c|}
\hline \multirow[b]{2}{*}{ Toxicity } & \multicolumn{2}{|c|}{ EF-IMRT, n (\%) } & \multicolumn{2}{|c|}{ Pelvic IMRT, n (\%) } & \multirow[b]{2}{*}{ P-value } \\
\hline & Grade 3 & Grade 4 & Grade 3 & Grade 4 & \\
\hline Gastrointestinal & & & & & $0.44^{\mathrm{a}}$ \\
\hline Upper GI & $2(3.6)$ & 0 & 0 & 0 & $0.50^{\mathrm{a}}$ \\
\hline Lower GI & $3(5.5)$ & 0 & $2(3.8)$ & 0 & $0.439^{\mathrm{a}}$ \\
\hline Hematological & & & & & 0.18 \\
\hline $\mathrm{Hgb} / \mathrm{Hct}$ & $4(7.3)$ & 0 & $2(3.8)$ & 0 & $0.68^{\mathrm{a}}$ \\
\hline WBC & $8(14.5)$ & $2(3.6)$ & $5(9.6)$ & $0(0.0)$ & 0.27 \\
\hline Neutrophils & 7 (12.7) & $2(3.6)$ & $4(7.7)$ & $1(1.9)$ & 0.58 \\
\hline Platelets & 0 & 0 & 0 & 0 & \\
\hline Genitourinary & 0 & 0 & 0 & 0 & \\
\hline
\end{tabular}

${ }^{a}$ Fisher's exact test. GI, gastrointestinal; Hct, hematocrit; Hgb, hemoglobin; WBC, white blood cell.

studies, EBRT was delivered to the pelvis and para-aortic regions using four-field or antero-posterior/postero-anterior (AP/PA) field techniques $(12,13)$. The inferior survival rate was attributed to the limitation of conventional radiotherapy in dose escalation to positive periaortic nodes due to concerns of toxicity to adjacent critical structures (14). According to a previous dosimetric publication, grossly involved PALNs may be treated with a simultaneous integrated boost (SIB) to 60 Gy while limiting dose to the small bowel, bone marrow and kidney using IMRT (15). Use of a SIB results in a decrease in overall treatment time, limiting tumor repopulation, with delivery of an increased dose per fraction resulting 
in an increased biologically equivalent dose and potentially resulting in a higher rate of local control (16).

As presented in Table I, pretreatment characteristics were well-balanced across the two treatment groups, with the exception of age and the extent of lymph node involvement (common iliac nodes or PALN vs. negative or lower pelvic nodes). Lymph node status in patients with cervical cancer is an important determinant of prognosis. Furthermore, the most distant level of lymph node involvement influenced the cervical cancer survival outcome (17). As such, an increased level of lymph node involvement predicts poorer survival, which support the univariate analysis results of the present study. Therefore, patients in the EF-IMRT group of the present study were hypothesized to present with poorer survival rates than patients in the pelvic IMRT group if the same irradiation volume of external beam radiotherapy was delivered. However, these OS and DFS rates were not significantly different between the two groups $(\mathrm{P}>0.05)$, and Cox's regression analysis indicated that EFirradiation was a protective prognostic factor for OS and DFS time, demonstrating that EF-IMRT is an efficient treatment for patients with uterine cervical cancer with involved common iliac nodes or PALNs to eradicate lymphatic micrometastasis and cure grossly involved PALNs.

Locoregional failure is known to be the predominant site of failure in patients treated with curative intent using radiotherapy alone (18), and a markedly increased rate of distant failure was previously documented in patients treated with concurrent chemoradiotherapy (11). However, in the present study, the locoregional failure rate was increased compared with the distant failure rate, potentially due to the fact that: i) Only 72 patients received concurrent chemotherapy, which may sensitize tumor cells to radiation, further enhance shrinkage of the primary tumor and achieve favorable locoregional control; ii) the present study had a shorter duration of follow-up; iii) para-aortic node relapse was defined as distant failure in previous studies and defined as regional failure in the present study; and iv) the size criterion for detecting LN of $>1 \mathrm{~cm}$ was employed, which may fail to deliver a boosted dose to the involved nodes with a short diameter of $<1 \mathrm{~cm}$, and lead to increased regional failure rates.

Kidd et al (3) reviewed the clinical data of 560 patients with cervical cancer who underwent pretreatment PET/CT staging and concluded that the risk of recurrence increased incrementally on the basis of the most distant level of nodal involvement, with a hazard ratio of 2.40 for pelvic nodes and 5.88 for para-aortic nodes. However, the failure rate and the proportions of failure sites did not indicate a difference between the two groups in the present study, further illustrating that EF-IMRT may decrease the risk of recurrence for patients with an increased level of lymphatic involvement.

With conventional radiotherapy techniques, generous portions of the small bowel and vertebrae are included in the treatment field, resulting in markedly increased gastrointestinal and hematological toxicities, as well as treatment interruption and severe late toxicities. According to Yoon et al (11), grade 3 or 4 acute toxicities were observed in $42 \%$ of 90 patients treated with AP/PA EF irradiation. Furthermore, 38\% of patients treated with four-field EFRT, in a study conducted by Rajasooriyar et al (13), experienced overall grade 3 or 4 acute toxicities. In comparison, grade 3 acute toxicity was experienced by $34.5 \%$ of the patients treated with EF-IMRT in the current study; a superior result to those demonstrated in previous studies. IMRT use assisted in the conformity of dose distribution, confined the high-dose portions of radiation fields, and decreased the absorbed dose and volume in critical organs, resulting in decreased overall toxicity. Gerszten et al (19) identified a significant decrease in critical organ irradiation following EF-IMRT treatment and subsequently suggested that this treatment may decrease acute and late treatment-associated side effects.

The target volume and the irradiated volume of vertebrae and small bowel tend to be considerably increased for EF irradiation than that for pelvic irradiation. Thus, the rate and severity of acute and late toxicities are expected to increase for EFRT. Although IMRT, as a means to decrease toxicities, was applied in the present study, the rate of acute toxicities increased within a tolerated range for EF-IMRT. Beriwal et al (20) reported that $1 / 3$ of the patients treated with EF-IMRT exhibited grade 3 or higher acute toxicities. These results are comparable with the results of the present study. The use of IMRT assisted in the conformation of the radiation dose and thus decreased the exposure of the small bowel and marrow, resulting in decreased toxicity.

In conclusion, para-aortic irradiation with IMRT may improve survival and alter the recurrence patterns of patients with involved common iliac nodes or PALNs who tended to experience poorer survival and an increased risk of recurrence, but were similar to those results of patients with or without lower pelvic nodal metastasis. Furthermore, with regard to EF-IMRT, the rate of acute toxicities increased, but remained within the acceptable range in comparison with that of pelvic IMRT. Overall, EF irradiation with IMRT should be electively performed on patients with cervical cancer with common iliac or periaortic nodal involvement to improve survival.

\section{References}

1. Lin MY, Jobling TW and Narayan K: Surgical staging of para-aortic LN in patients with locally advanced cervix cancer and no evidence of metastases in preoperative PET/CT imaging. J Gynecol Oncol 26: 352-354, 2015.

2. FIGO Committee on Gynecologic Oncology: FIGO staging for carcinoma of the vulva, cervix, and corpus uteri. Int J Gynaecol Obstet 125: 97-98, 2014

3. Kidd EA, Siegel BA, Dehdashti F, Rader JS, Mutch DG, Powell MA and Grigsby PW: Lymph node staging by positron emission tomography in cervical cancer: Relationship to prognosis. J Clin Oncol 28: 2108-2113, 2010.

4. Rotman M, Pajak TF, Choi K, Clery M, Marcial V, Grigsby PW, Cooper J and John M: Prophylactic extended-field irradiation of para-aortic lymph nodes in stages IIB and bulky IB and IIA cervical carcinomas. Ten-year treatment results of RTOG 79-20. JAMA 274: 387-393, 1995.

5. Gay HA, Barthold HJ, O'Meara E, Bosch WR, El Naqa I, Al-Lozi R, Rosenthal SA, Lawton C, Lee WR, Sandler H, et al: Pelvic normal tissue contouring guidelines for radiation therapy: A radiation therapy oncology group consensus panel atlas. Int $\mathrm{J}$ Radiat Oncol Biol Phys 83: e353-e362, 2012.

6. Lim K, Small W Jr, Portelance L, Creutzberg C, Jürgenliemk-Schulz IM, Mundt A, Mell LK, Mayr N, Viswanathan A, Jhingran A, et al: Consensus guidelines for delineation of clinical target volume for intensity-modulated pelvic radiotherapy for the definitive treatment of cervix cancer. Int J Radiat Oncol Biol Phys 79: 348-355, 2011. 
7. Kabolizadeh P, Fulay S and Beriwal S: Are radiation therapy oncology group para-aortic contouring guidelines for pancreatic neoplasm applicable to other malignancies-assessment of nodal distribution in gynecological malignancies. Int J Radiat Oncol Biol Phys 87: 106-110, 2013.

8. Oken MM, Creech RH, Tormey DC, Horton J, Davis TE, McFadden ET and Carbone PP: Toxicity and response criteria of the eastern cooperative oncology group. Am J Clin Oncol 5 : 649-655, 1982

9. Cox JD, Stetz J and Pajak TF: Toxicity criteria of the radiation therapy oncology group (RTOG) and the European organization for research and treatment of cancer (EORTC). Int J Radiat Oncol Biol Phys 31: 1341-1346, 1995.

10. Salama JK, Mundt AJ, Roeske J and Mehta N: Preliminary outcome and toxicity report of extended-field, intensity-modulated radiation therapy for gynecologic malignancies. Int J Radiat Oncol Biol Phys 65: 1170-1176, 2006.

11. Yoon HI, Cha J, Keum KC, Lee HY, Nam EJ, Kim SW, Kim S, Kim YT, Kim GE and Kim YB: Treatment outcomes of extended-field radiation therapy and the effect of concurrent chemotherapy on uterine cervical cancer with para-aortic lymph node metastasis. Radiat Oncol 10: 18, 2015.

12. Wu SY, Huang EY, Chanchien CC, Lin H, Wang CJ, Sun LM, Chen HC, Fang FM, Hsu HC and Huang YJ: Prognostic factors associated with radiotherapy for cervical cancer with computed tomography-detected para-aortic lymph node metastasis. J Radiat Res 55: 129-138, 2014.

13. Rajasooriyar C, Van Dyk S, Bernshaw D, KondalsamyChennakesavan S, Barkati M and Narayan K: Patterns of failure and treatment-related toxicity in advanced cervical cancer patients treated using extended field radiotherapy with curative intent. Int J Radiat Oncol Biol Phys 80: 422-428, 2011.
14. Coulombe G, Thiessen B, Balkwill S and Aquino-Parsons C: Polyradiculopathy post-concomitant chemoradiation for carcinoma of the uterine cervix treated with pelvic and para-aortic fields. Gynecol Oncol 99: 774-777, 2005.

15. Ahmed RS, Kim RY, Duan J, Meleth S, De Los Santos JF and Fiveash JB: IMRT dose escalation for positive para-aortic lymph nodes in patients with locally advanced cervical cancer while reducing dose to bone marrow and other organs at risk. Int J Radiat Oncol Biol Phys 60: 505-512, 2004.

16. Boyle J, Craciunescu O, Steffey B, Cai J and Chino J: Methods, safety and early clinical outcomes of dose escalation using simultaneous integrated and sequential boosts in patients with locally advanced gynecologic malignancies. Gynecol Oncol 135: 239-243, 2014.

17. Grigsby PW, Siegel BA and Dehdashti F: Lymph node staging by positron emission tomography in patients with carcinoma of the cervix. J Clin Oncol 19: 3745-3749, 2001.

18. Sakurai H, Mitsuhashi N, Takahashi M, Akimoto $\mathrm{T}$, Muramatsu H, Ishikawa H, Imai R, Yamakawa M, Hasegawa M and Niibe H: Analysis of recurrence of squamous cell carcinoma of the uterine cervix after definitive radiation therapy alone: Patterns of recurrence, latent periods, and prognosis. Int J Radiat Oncol Biol Phys 50: 1136-1144, 2001.

19. Gerszten K, Colonello K, Heron DE, Lalonde RJ, Fitian ID, Comerci JT, Selvaraj RN and Varlotto JM: Feasibility of concurrent cisplatin and extended field radiation therapy (EFRT) using intensity-modulated radiotherapy (IMRT) for carcinoma of the cervix. Gynecol Oncol 102: 182-188, 2006.

20. Beriwal S, Gan GN, Heron DE, Selvaraj RN, Kim H, Lalonde R, Kelley JL and Edwards RP: Early clinical outcome with concurrent chemotherapy and extended-field, intensity-modulated radiotherapy for cervical cancer. Int J Radiat Oncol Biol Phys 68: 166-171, 2007. 